\title{
Transport Safety Mechanism of School Children Using IOT based Smart System
}

\author{
Nada Abdul Al-Balushi \\ Syed Imran Ali Kazmi \\ Faisal Khalifa Al-Kalbani
}

\author{
Middle East College \\ Middle East College \\ Middle East College
}

\begin{abstract}
Most of the Students in Sultanate of Oman are using bus to reach their schools. Bus considered as one of the convenient way of traveling. As most of Students are traveling from home to school or vice versa by bus. Safety is the biggest concern and issue. Bus driver and parents are responsible for providing safety and security to the schoolchildren's. Many of children went to school and never returned home afterwards. This is because the child feels tired and then sleeps after a hard school day. The bus driver did not pay attention to the presence of a sleeping child in the school bus and the bus driver closes the windows and the door, causing suffocation and death. There are also a lot of accidents that might happen on school buses, so researcher are working to find the way of tracking school bus which is very important for the monitoring of location and track students throughout journey from and to school. This research paper presents IOT smart transportation system for a children school. The system consists of IR sensors to calculate the number of students, RFID card and RFID reader to read student data and attendance recording as well as MQ3 sensor for alcohol sensing and to ensure the safety of the driver. It also contains a mobile application to get notifications and messages and shows other information such as the time of riding the student on the school bus and coming off the bus you can also take the latest coordinates and thus follow the bus via Google Map.
\end{abstract}

\section{Introduction}

The population is increasing in all countries of the world as well as in the Sultanate of Oman. Therefore an increase in the number of vehicles and transportation. It is necessary to have new transport systems to reduce traffic accidents. Increasing the population means an increase in the number of children who attend school every year.

Providing safety for schoolchildren is the responsibility of the parents, and the school administration as well as the bus driver. The parents must teach the child how to protect themselves from the risks they may face on their journey to and from school. It is the school's responsibility to provide new school bus systems to keep children safe. The bus driver must comply with the speed rules when transporting children to and from school, he must also be fully aware and not drink alcohol so as not to cause traffic accidents. Many accidents occurred on school buses and resulted in the deaths of children. One such accident resulted in the death of a four-year-old boy who left alone on the school bus for long hours. This accident occurred in 2016 in Al Ma'abila. The 4-year-old boy was sleeping in the back seat of the bus and forgot there. After the child forgotten on the bus more than 4 hours, the child found dead due to suffocation(Mukrashi, 2016).

Another accident occurred in the Rui area where an 8-year-old Sudanese child died. Because the bus driver did not inspect the bus before the bus closed, there are no children on the bus. The child died after forgotten on the school bus because of suffocation(Times, 2018).

There are many and many accidents that have occurred in school buses and the reasons for these 


\section{Journal of Student Research}

Fourth Middle East College Student Research Conference, Muscat, Sultanate of Oman

accidents are the lack of an intelligent system for school buses, Lack of network in many schools in Oman and most of all, children have no skills in how to protect themselves when a problem occurs on the school bus.

This paper aims to build smart transportation system for school buses to reduce the occurrence of suffocation and death that occurs in school buses because of forgetting the child on the closed bus for long hours when the child feels asleep and the bus driver did not pay attention the presence of a child on the bus.

The paper also discusses how to use the Internet of Things to reduce accidents, forgetting children in school buses through a smart and integrated system. The system consists of sensors that are used to sense schoolchildren's when riding a school bus. Where it calculates the number of students when they ride the bus, which called IR sensors. It also consists of alcohol sensors as I mentioned earlier. In addition, it consists of RFID reading device that have been used to read students' data and then text messages are sent to parents as well as the school administration or send notifications in the mobile application. The data transmitted through a microcontroller called Node MCU, which transmits information from the sensor to the cloud through Wi-Fi.

A mobile application that connects to the cloud is use to display all information from the sensor and display it to the end user after processing. Thus providing a safer system for children and reducing suffocation accidents in school buses. In addition, through the application parents and school administrators can track the location of the bus by using Google Map.

Many models can used to solve accidents of forgetting children in school buses and are one of the biggest problems that may occur in the school environment and result in loss of life. Through the Internet of Things, we are looking for solutions to this problem, and rather we are looking for the best system with high efficiency and speed at the lowest cost. Moreover, the system can be select and modified by the companies as required.

Thing Speck is an Internet of Things cloud platform that has been use in this system. This platform does not require licenses. Receives data from sensors, takes time stamps and then outputs them to users' devices. Any organization can use the public cloud by paying all the costs of computing resources. The public cloud support many users and managed by external organizations. The infrastructure is also at the provider's premises, not users. The operating systems or databases provided by public cloud service providers are limited (Group, 2016).

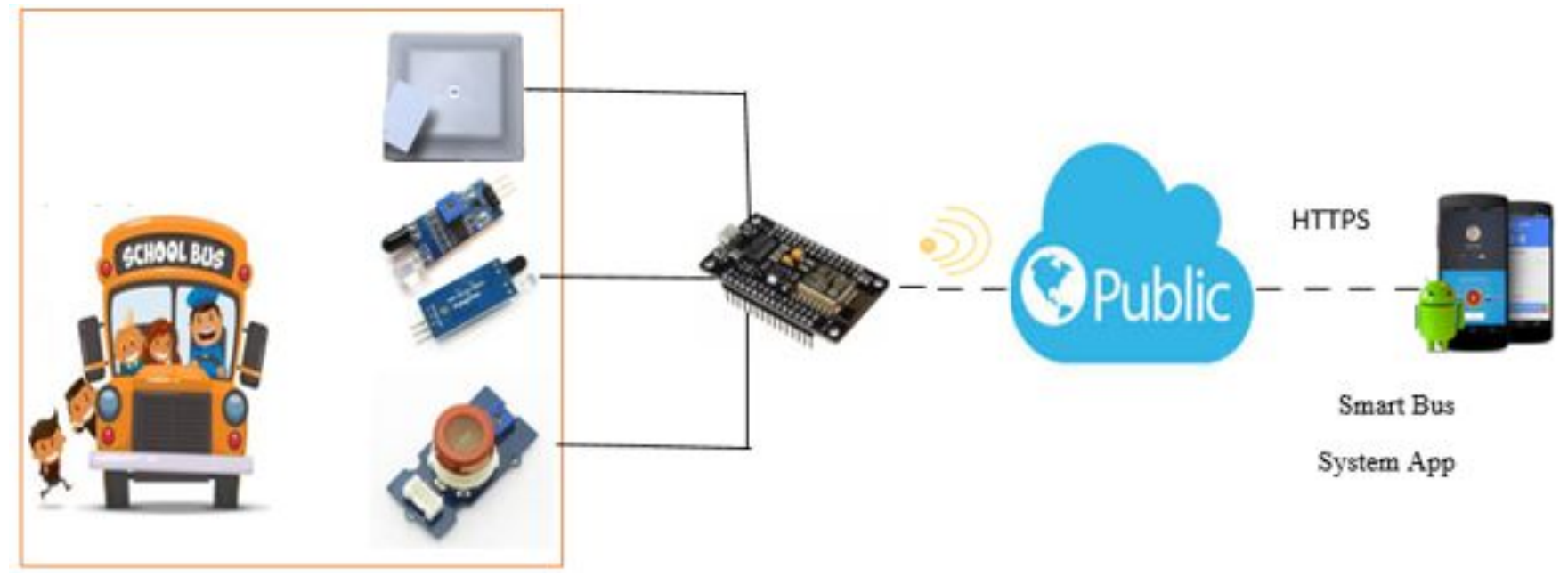

Figure 1. System design (Public cloud)

\section{Literature review}




\section{Journal of Student Research}

Fourth Middle East College Student Research Conference, Muscat, Sultanate of Oman

This part includes the ideas to provide a clear understanding about what going to do in this system design of safe transportation. Moreover, it includes an appropriate explanation of the technologies used in this research paper.

\section{Internet of things (IoT)}

According to Prokodi et al (Porkodi, 2014)article, Internet of things makes the objects themselves identifiable, gain intelligence, convey information about themselves and can access information gathered by other things. In addition, the Internet of Things allows you to communicate with people and things anytime, anywhere, with anyone, any route/network, and any service. This means handles elements such as content, convergence, computing, and communication. Internet of Things become established in cities, transport, buildings, smart factories and in many other areas even, a street light being networked. The aim of IoT is to create intelligent environments, makes things smart and allowing objects to connect at anytime and anywhere with any things (Ovidiu Vermesan, 2011).

Another article titled 'Introduction on IoT' (Bhat, 2018)it was said that the network consists of physical objects embedded with sensors and network connectivity, through which these objects can collect and share data. As well as sensing objects and remote control over the current network infrastructure, like the internet of things has become a reason for improving efficiency and accuracy in the direct integration of physical objects (Bhat, 2018).

\section{RFID tag}
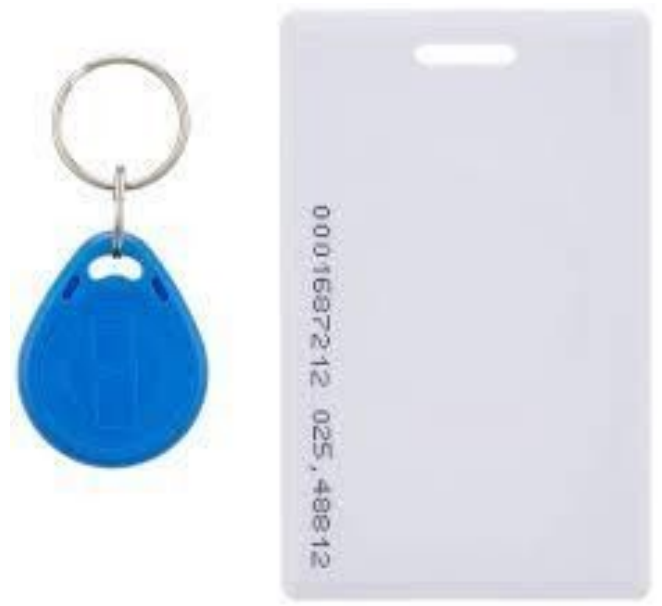

Figure 2. RFID tag

The RFID tag is a small electronic device containing a microchip for storage and calculation and a coupling element such as an antenna file to communication (DAVINDER PARKASH, 2012). The information collected done by chip and transmitted wirelessly. The RFID tag categorized into different categories such as Active Tag, Passive Tag and semi-passive (DAVINDER PARKASH, 2012).

\section{MQ3 Sensor}

This sensor used to sense alcohol. It has a high sensitivity to alcohol and small sensitivity to benzene. The potentiometer sensitive material of MQ3 gas sensor is $\mathrm{SnO}$, which with lower conductivity in clean air can adjust the sensitivity. When the target alcohol gas exist, the sensors conductivity is higher along with the gas concentration rising. It has fine sensitivity range around 2 meters (Pranjali Ingalepatil, 2017). 
Figure 3. $M Q 3$ sensor

\section{Microcontroller (Node MCU)}

Is a circuit of open-source programming. And a Wi-Fi chip that has 11 GPIO input and output pins. It includes an accurate console as well as a full TCP / IP stack. Moreover, it is combining some features of the Arduino board (Ali Al Dahoud, 2018).

\section{Methodology}

PPDIOO Method is integrated with research qualitative methodology. PPDIOO is a cisco methodology determines the lifecycle of the services required for a network. As it show in in figure4, this methodology consists of six phases: Prepare Plan, Design, Implement, Operate and Optimize (Balaji Sivasubramanian, 2010). Prepare includes the establishment of regulatory requirements, the development of a network strategy and the proposal for a high-level conceptual architecture that identifies techniques that can better support the architecture. This phase can create a finance justification for the network strategy (Balaji Sivasubramanian, 2010). Plan includes the identification of initial network requirements based on objectives, facilities, user needs, etc. The Research methodology plan is useful to help manage the tasks, responsibilities, milestones and resources needed to implement changes to the network. In addition, research-based development plan must be in line with the scope, cost, and resource parameters specified in the original business requirements (Balaji Sivasubramanian, 2010). A design that complies with business objectives and technical requirements can improving network performance while supporting high availability, reliability, security and scalability (Semperboni, 2009). Implement Building a network is essential for any successful organization and must provide vital services without interruption. In the implement stage, the company integrates new devices and capabilities according to design without compromising network availability or performance (Balaji Sivasubramanian, 2010). Operate this phase involves maintaining network health through daily operations. As well, as maintain availability and reduce costs as it provides error detection, correction and performance control (Balaji Sivasubramanian, 2010). Optimize includes proactive network management. Its purpose is to identify and solve problems before they affect the organization. In the PPDIOO methodology, the optimization phase can be call for network redesign in case of many network problems and errors (Balaji Sivasubramanian, 2010). The network designer should follow a methodology that guides the entire life cycle of the design process. In this regard, integrated PPDIOO methodology for research plan and qualitative method. In this research data collection by gathering information, clear understanding the problem, and find solutions for this problem. The goal is to know the processes and behaviors of the proposed system and the services that the proposed system will provide, with the help of information that collects the function of the proposed system known, and the services and techniques that must be improve for the new system are known. Specific plans are also create and approved for project start-ups. To design a new network with new requirements, PPDIOO methodology is used to help reduce cost while establishing and improving the network. WBS is use in this project to distribute the tasks that will be use in the methodology chosen and to divide the tasks into smaller tasks. The researched paper is being organized and achieve success.

Figure 4. PPDIOO lifecycle approach model

\section{Purposed System}

IOT base smart bus transportation system proposed to ensure the safety of children along with entering or leaving of children from the school bus. Each child has an RFID tag on his or her smart card that helps for identifying the child. Children identified based on the information, which is stored in the RFID tag itself. Moreover, this information will exchange over a radio waves. When 
the child enters or exits the school bus, the RFID reader, which is install in the school bus, will record a response and this information is pressed for further implementation. Smart bus transportation system is also informing parents when their children entering or exiting the school bus by sending SMS for them. In addition, the school management will receive a SMS of each child travelling. The number of children riding the bus counted through IR IN-OUT sensors this will ensure that the school bus is empty or still their children inside the bus. Where the tracking system is use to know the location and route of the bus, and can be analyze from any remote location using GPS technology and Google maps. Finally, MQ3 sensor used in this smart system to reduce road accidents related to drunk driving. MQ3 sensor senses alcohol molecules in the air breathe by the drive. Where this sensor connected to microcontroller (Node MCU), the received data from MQ3 sensor will be send it to microcontroller. The analog output of which applied to the Arduino board it send it wirelessly to server and the information will read it by Arduino Software (IDE) were the reference variants of Arduino.

Figure 5. Block Diagram of the Purposed System

\section{Framework}

This framework show the cycle of IOT smart transportation system. For the system to runs successfully, all the sub systems need to be connected. The sensitive devices (IR sensors and alcohol sensor) that located in the bus connected to the microcontroller (Node MCU) and programmed by using Arduino software, where this program used for code the sensor and connectivity devices to working with each other. The data will send from the microcontroller to cloud through Wi-Fi by using the HTTPS protocol. The location information will be send from Node MCU by GPS modem through satellite to the cloud. All the received data will filtered and analyzed in the server and the results will send it to the mobile application using HTTPS protocol when the IR sensors detect the presence of any child when riding a school bus it will send the data directly to the cloud by the Node MCU. Finally, this application also provides the location of the bus by taking the latest coordinates and follow it by using google map.

Figure 6. Framework based IoT smart transportation system

\section{Results of proposed method}

The benefits that have been add in the proposed method will be beneficial to the school and the community as well. The proposed method has many benefits such as increased safety and security in the school environment. By tracking the road minute by minute from the parents on the bus and the school administration, they can make sure they know when they send their children to the bus and when they will be delivering when they leave the bus. In addition, this research will provide real-time data to allow a fast response to emergencies.

\section{Discussion}

This following figure shows the devices connected to cloud computing and how they work. Starting from the bus unit, the sensors on the school bus connected to the Node MCU that connects to the cloud via Wi-Fi. Moreover, the cloud has all the data. Provides services that help send information to the mobile application using HTTPS. In addition, there is a reader in the school bus unit, when the child/ student scans RFID card in to RFID reader the reader will read the student information. Moreover, send this information through microcontroller to the cloud where the database server search for a particular ID and send the detailed information of that student to the mobile 


\section{Journal of Student Research}

Fourth Middle East College Student Research Conference, Muscat, Sultanate of Oman

application, with the time duration when the student rides the bus until the student arrives school and get off the bus. Then again ride the bus from school until arriving back home.

Figure 7. Work of devices connecting to cloud computing

The next figure shows the test bed for IoT smart transportation system.

Figure 8. Test Bed

\section{Aim of a research paper}

Develop a smart transportation system for schoolchildren in sultanate of Oman for providing a secure and safe environment with high availability and tracking ability.

\section{Conclusion}

In conclusion, we conclude that through the implementation of this research can reduce the suffocation accidents in school buses facing most of the children's schools and the smart system will ensure the safety of students during pick up and drop off. . This research will be beneficial for both schools and the community. A reliable network designed, as it is manageable and scalable to become more accurate in the future.

\section{References}

Ali Al Dahoud, M. F. (2018). NodeMCU V3 For Fast IoT Application Development.

Balaji Sivasubramanian, E. F. (2010, July 15). CISCO. Retrieved from ciscopress.com: http://www.ciscopress.com/articles/article.asp?p=1608131\&seqNum=3

Bhat, O. (2018). Introduction to IOT . International Advanced Research Journal in Science, Engineering and Technology, 5(1), 41-43.

DAVINDER PARKASH, T. K. (2012, September). THE RFID TECHNOLOGY AND ITS APPLICATIONS: A REVIEW . nternational Journal of Electronics, Communication \& Instrumentation Engineering Research and Development, pp. 111-113.

Group, W. B. (2016). Cloud Computing Overview.

Marcello A. Gómez Maureira, D. O. (2014). ThingSpeak - an API and Web Service for the Internet of Things.

Mukrashi, F. A. (2016). Kindergarten boy dies after being left in Oman school bus. Oman: Gulf News.

Ovidiu Vermesan, P. F. (2011, March 1). Internet of Things beyond the Hype: Research, Innovation and Deployment. Internet of Thinge Vision, pp. 15-17.

Porkodi, D. V. (2014, March). The Internet of Things (IoT) Applications and Communication Enabling Technology Standards: An Overview. 2014 International Conference on Intelligent Computing Applications, p. 324. 
Pranjali Ingalepatil, e. a. (2017). Alcohol Detection System in Vehicle Using Arduino. International Research Journal of Engineering and Technology (IRJET) , 4(6), 288.

Semperboni, F. (2009, junwary 29). Retrieved from CiscoZine.com: https://www.ciscozine.com/theppdioo-network-lifecycle/

Times, N. (2018, December 30). Safety system to be launched in Oman school buses. Muscat, Oman. 